\title{
Effect of corticosteroids on human osteoclast formation and activity
}

\author{
T Hirayama, A Sabokbar and N A Athanasou
}

Department of Pathology, Nuffield Department of Orthopaedic Surgery, University of Oxford, Nuffield Orthopaedic Centre, Oxford OX3 7LD, UK

(Requests for offprints should be addressed to N A Athanasou; Email: nick.athanasou@ndos.ox.ac.uk)

\begin{abstract}
Chronic corticosteroid treatment is known to induce bone loss and osteoporosis. Osteoclasts are specialised boneresorbing cells that are formed from mononuclear phagocyte precursors that circulate in the monocyte fraction. In this study we have examined the effect of the synthetic glucocorticoid, dexamethasone, on human osteoclast formation and bone-resorbing activity. Human monocytes were cultured for up to 21 days on glass coverslips and dentine slices, with soluble receptor activator for nuclear factor $\kappa \mathrm{B}$ ligand (RANKL; $30 \mathrm{ng} / \mathrm{ml}$ ) and human macrophage-colony stimulating factor (M-CSF; $25 \mathrm{ng} / \mathrm{ml}$ ) in the presence and absence of dexamethasone $\left(10^{-8} \mathrm{M}\right)$. The addition of dexamethasone over a period of 7 and 14 days of culture of monocytes (during which cell proliferation and differentiation predominantly occurred) resulted
\end{abstract}

in a marked increase in the formation of tartrate-resistant acid phosphatase-positive multinucleated cells and an increase in lacunar resorption. The addition of dexamethasone to monocyte cultures after 14 days (when resorptive activity of osteoclasts had commenced) reduced the extent of lacunar resorption compared with cultures to which no dexamethasone had been added. The addition of dexamethasone to osteoclasts isolated from giant cell tumours of bone significantly inhibited resorption pit formation. Our findings indicate that dexamethasone has a direct effect on osteoclast formation and activity, stimulating the proliferation and differentiation of human osteoclast precursors and inhibiting the bone-resorbing activity of mature osteoclasts.

Journal of Endocrinology (2002) 175, 155-163

\section{Introduction}

Corticosteroid-induced bone loss is the most common form of secondary osteoporosis. It is usually the result of excessive treatment with corticosteroids and results in loss of cortical and cancellous bone and an increased risk of pathological fracture (Adachi 1997, Eastell et al. 1998, Van Staa et al. 2000). Osteoporosis secondary to corticosteroid treatment is believed to result from effects on the bone remodelling unit, with osteoblastic bone formation and osteoclastic resorption being affected (Nishimura \& Ikuyama 2000). Bone loss after corticosteroid treatment is characterised by an initial phase of rapid bone resorption followed by a more chronic phase in which bone is lost gradually (Bressot et al. 1979, Dempster 1989); it has been noted that there is an increase in osteoclast numbers and sites of lacunar resorption in the bones of patients receiving steroid treatment. This increase in osteoclast numbers is thought to be due to either increased formation of osteoclasts or increased osteoclast survival (Manolagas 2000).

Osteoclasts are formed by fusion of bone marrowderived precursors that circulate in the monocyte fraction of peripheral blood (Fujikawa et al. 1996). Osteoclast formation requires the presence of macrophage-colony stimulating factor (M-CSF) and involves interaction between $\mathrm{CD} 14^{+}$monocytes, which express the receptor activator of nuclear factor (NF)- $\kappa B$ (RANK), and RANK ligand (RANKL), which is expressed by osteoblasts (Tanaka et al. 1993, Matsuzaki et al. 1998, Quinn et al. 1998, Yasuda et al. 1998). Although the precise manner in which corticosteroids stimulate osteoclast formation is unclear, these compounds are known to enhance osteoclast formation from marrow precursors in vitro and to increase and decrease respectively osteoblast expression of RANKL and osteoprotegerin, the soluble decoy receptor for RANK (Hofbauer et al. 1999). The effect of corticosteroids on osteoclast bone-resorbing activity is controversial, with both enhancement and inhibition of the activity of bone-resorbing cells being reported (Raisz et al. 1972, Teitelbaum et al. 1981, Reid et al. 1986, Tobias \& Chambers 1989). Corticosteroids are also believed to influence osteoclastic bone resorption by enhancing osteoclast apoptosis (Tobias \& Chambers 1989, Dempster et al. 1997).

In this study, we have sought to analyse the effect of corticosteroids on human osteoclast formation and activity. We have defined the proliferation and activation stages of human osteoclast formation and, in this way, determined the effect of the synthetic glucocorticoid, dexamethasone, on the formation and resorbing activity of human osteoclasts formed from circulating precursors. In addition, we 
have analysed the effect of dexamethasone on lacunar resorption by mature osteoclasts. Our findings indicate that dexamethasone stimulates the proliferation and differentiation of osteoclast precursors and inhibits lacunar resorption by mature osteoclasts isolated from giant cell tumours of the bone.

\section{Materials and Methods}

\section{Materials}

All cell incubations were performed in $\alpha$ minimal essential medium (MEM) (Gibco, UK) supplemented with glutamine $(2 \mathrm{mM})$, benzyl penicillin $(100 \mathrm{IU} / \mathrm{ml})$, streptomycin $(100 \mu \mathrm{g} / \mathrm{ml})$ and $10 \%$ heat-inactivated fetal calf serum (FCS) (MEM/FCS; Gibco, UK) in a humidified atmosphere of $5 \% \mathrm{CO}_{2}$ at $37{ }^{\circ} \mathrm{C}$. Dexamethasone (Sigma, UK) was dissolved in absolute alcohol and stored at $-20{ }^{\circ} \mathrm{C}$. Human M-CSF (R\&D Systems Europe, Abingdon, Oxon, UK) and soluble (s)RANKL (Amgen Inc., Thousand Oaks, CA, USA) were dissolved in MEM/ FCS and stored at $-20^{\circ} \mathrm{C}$.

\section{Human osteoclast formation in vitro}

Monocytes were isolated from the peripheral blood of six normal men (age range 25-53 years) and six normal women (age range 31-58 years). After collection, blood was diluted 1:1 in MEM, layered over Ficoll-Hypaque (Pharmacia, UK), centrifuged at $693 \mathrm{~g}$ and washed and resuspended in MEM/FCS. The number of cells in the resulting suspension of peripheral blood mononuclear cells (PBMCs) was counted in a haemocytometer after lysis of red cells with a $5 \%(\mathrm{v} / \mathrm{v})$ acetic acid solution.

PBMCs isolated as detailed above were seeded at $5 \times 10^{5}$ cells per well into $7 \mathrm{~mm}$ wells of a 96-well tissue-culture plate containing either slices of dentine (4 $\mathrm{mm}$ diameter) or glass coverslips $(6 \mathrm{~mm}$ diameter). After $2 \mathrm{~h}$ incubation, dentine slices and coverslips were removed, washed in MEM and placed in 24-well tissueculture plates containing $1 \mathrm{ml}$ MEM/FCS with added sRANKL (30 ng/ml) and M-CSF (25 ng/ml). Cell cultures were incubated in the presence or absence of dexamethasone $\left(10^{-8} \mathrm{M}\right)$ for up to 21 days. Media and added factors were replaced entirely every 3-4 days. After incubation, the extent of osteoclast formation and of bone resorption were assessed as detailed below.

\section{Histochemical and immunohistochemical characterisation of cultured cells}

Histochemical staining for tartrate-resistant acid phosphatase (TRAP) was carried out using a commercially available kit (Sigma, UK). Cell preparations were fixed in citrate/acetone solution and stained for acid phosphatase, using naphthol AS-BI phosphate as a substrate, in the presence of $1.0 \mathrm{M}$ tartrate; the product was reacted with fast garnet GBC salt (Minkin 1982).

Cell preparations on coverslips were also stained immunohistochemically, using an indirect immunoperoxidase technique with the monoclonal antibody 23C6 (Serotec, Oxford, UK): this is directed against CD51, the vitronectin receptor (VNR), a highly osteoclast-associated antigen (Horton et al. 1985). Cell preparations were similarly stained with the monoclonal antibody GRS1, directed against CD14, a macrophage-associated antigen not known to be expressed by osteoclasts (Athanasou \& Quinn 1990). The number of $\mathrm{TRAP}^{+}$and $\mathrm{VNR}^{+}$multinucleated cells (MNCs) on each coverslip was counted in four fields of view $(10 \times$ objective $)$ and the mean taken; cells containing three or more nuclei were considered to be multinucleated.

\section{Functional evidence of osteoclast differentiation: detection of lacunar resorption}

Functional assessment of osteoclast formation was determined at the end of the culture period using a resorption assay. Dentine slices were placed in $\mathrm{NH}_{4} \mathrm{OH}(1 \mathrm{M})$ for $30 \mathrm{~min}$ and cleaned by ultrasonication to remove adherent cells (Boyde et al. 1984). The slices were then washed with distilled water, and stained with $0.5 \%(\mathrm{w} / \mathrm{v})$ toluidine blue for $3 \mathrm{~min}$ before being air-dried and examined by light microscopy. The surface of each dentine slice was examined by light microscopy for evidence of lacunar resorption. Using image analysis software (Photoshop 5.5, Adobe), the extent of resorption was determined by calculating the percentage surface area of lacunar resorption on each dentine slice.

Effect of dexamethasone on the proliferation and differentiation of human osteoclast precursors and lacunar resorption by mature osteoclasts

To determine the duration of the proliferative phase of osteoclast formation from circulating precursors, PBMC cultures were treated for various lengths of the incubation period with hydroxyurea (Sigma Chemicals) to inhibit DNA synthesis and cell proliferation in vitro (Tanaka et al. 1993). Hydroxyurea (1 mM) was added to coverslips and dentine slices containing PBMC cultures maintained in the presence of $30 \mathrm{ng} / \mathrm{ml} \mathrm{RANKL}$ and $25 \mathrm{ng} / \mathrm{ml} \mathrm{M-CSF}$. The effect on osteoclast generation was assessed in terms of the formation of $\mathrm{TRAP}^{+}$MNCs and evidence of lacunar resorption.

The effect of dexamethasone on cell proliferation was also evaluated by determining $\left[{ }^{3} \mathrm{H}\right]$ thymidine incorporation in acid-fast insoluble fractions of cultured cells. PBMCs $\left(1 \times 10^{6}\right.$ cells per well $)$ were cultured in $16-\mathrm{mm}$ wells containing $1 \mathrm{ml} \mathrm{MEM/FCS} \mathrm{in} \mathrm{the} \mathrm{presence} \mathrm{of} \mathrm{M-CSF,}$ RANKL and $10^{-8} \mathrm{M}$ dexamethasone. Radioisotope was added to cell cultures $24 \mathrm{~h}$ before the termination 

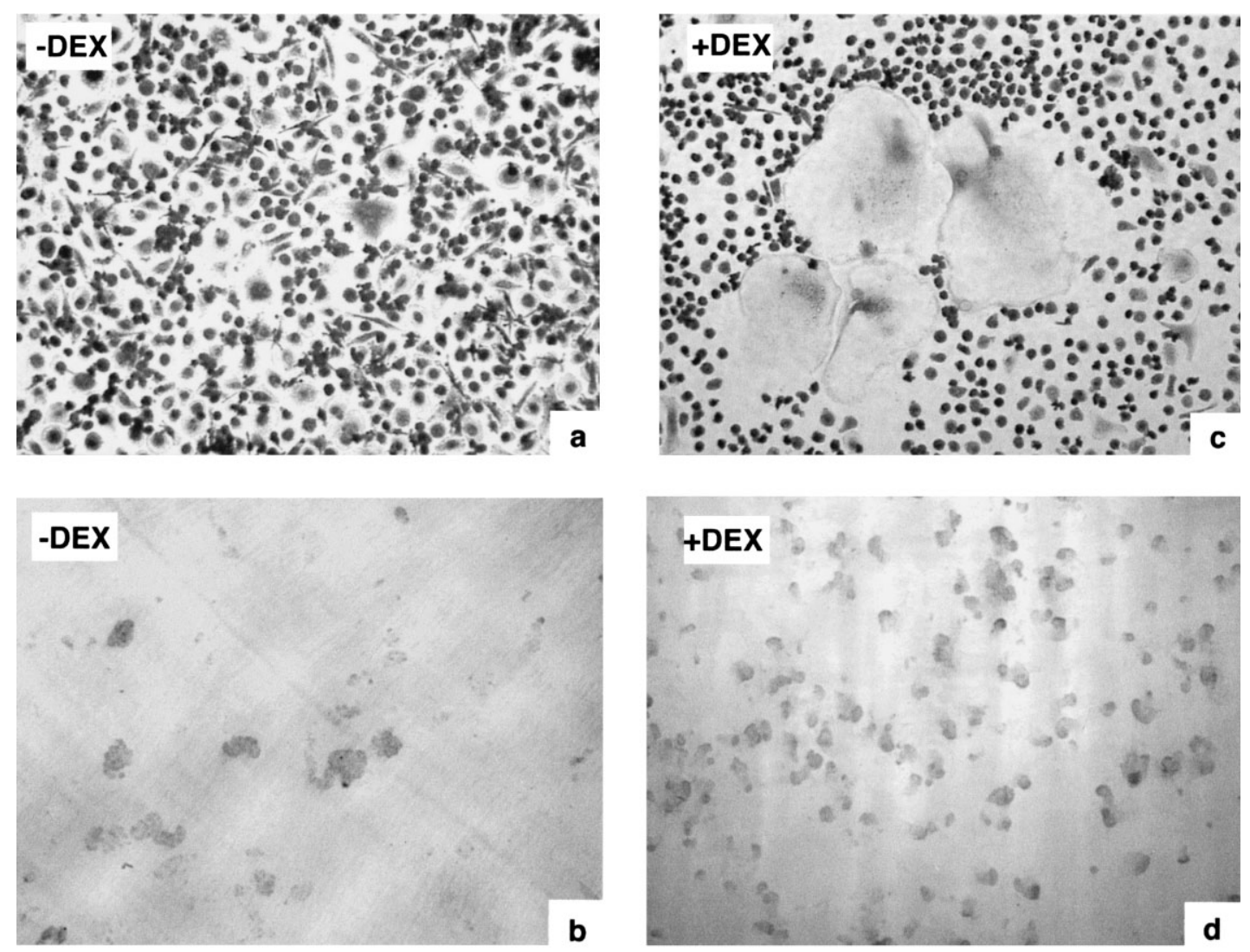

Figure 1 Osteoclast differentiation in PBMC cultures incubated with sRANKL (30 ng/ml) and M-CSF (25 ng/ml) as demonstrated by formation of TRAP ${ }^{+}$MNCs ((a) and (c); original magnification: $\left.\times 100\right)$ and lacunar resorption pit formation on dentine slices $((b)$ and $(d)$; original magnification: $\times 40)$ in the presence $(+)$ or absence $(-)$ of $10^{-8} \mathrm{M}$ dexamethasone (DEX).

of the experiments - that is, after 2, 3, 4, 5, 7 and 10 days in culture. Cells were lysed by the addition of trypsin and the radioactivity incorporated into trichloroacetic acid-

Table 1 Proliferative activity of osteoclast precursors in PBMC cultures, as demonstrated by lacunar resorption

\section{Treatment (at days)}

\begin{tabular}{|c|c|c|c|c|c|c|}
\hline $0-3$ & $3-7$ & $7-10$ & $10-14$ & $14-17$ & $17-21$ & (\% of control) \\
\hline- & - & - & - & - & - & 100 \\
\hline+ & + & + & + & + & + & 0 \\
\hline- & + & + & + & + & + & 0 \\
\hline- & - & + & + & + & + & 0 \\
\hline- & - & - & + & + & + & $62 \cdot 8$ \\
\hline- & - & - & - & + & + & $68 \cdot 4$ \\
\hline- & - & - & - & - & + & $101 \cdot 6$ \\
\hline
\end{tabular}

Human monocyte cell cultures were treated with $1 \mathrm{mM}$ hydroxyurea $(+)$ at various time points during the 21-day incubation. All cocultures were maintained in the presence of $30 \mathrm{ng} / \mathrm{ml}$ sRANKL and $25 \mathrm{ng} / \mathrm{ml} \mathrm{M-CSF}$ and $10^{-8} \mathrm{M}$ dexamethasone. The results are expressed as the percentage resorption compared with that in control cultures to which hydroxyurea was not added ( - ). insoluble fractions was then counted in a $\beta$-scintillation counter.

To examine the effect of corticosteroids on different stages of RANKL-induced osteoclast formation, dexamethasone $\left(10^{-8} \mathrm{M}\right)$ was added to PBMC cultures at various intervals over the 21 -day incubation period. The effect of adding dexamethasone on the formation of $\mathrm{TRAP}^{+}$MNCs on coverslips and lacunar resorption on dentine slices was assessed relative to control cultures that did not contain dexamethasone.

The effect of dexamethasone on lacunar bone resorption by mature osteoclasts obtained from two giant cell tumours of bone was also studied (Chambers et al. 1985). Each tumour was curetted with a scalpel and the resultant cell suspension added to dentine slices placed in 96well tissue culture plates. Cells were settled on the dentine slices for $2 \mathrm{~h}$, washed in MEM and then placed in 24-well tissue culture plates containing $1 \mathrm{ml} \mathrm{MEM}$ / FCS (in the presence or absence of $10^{-8} \mathrm{M}$ dexamethasone). Cell cultures were incubated for $24 \mathrm{~h}$, after which time the extent of lacunar resorption was assessed as detailed above. 


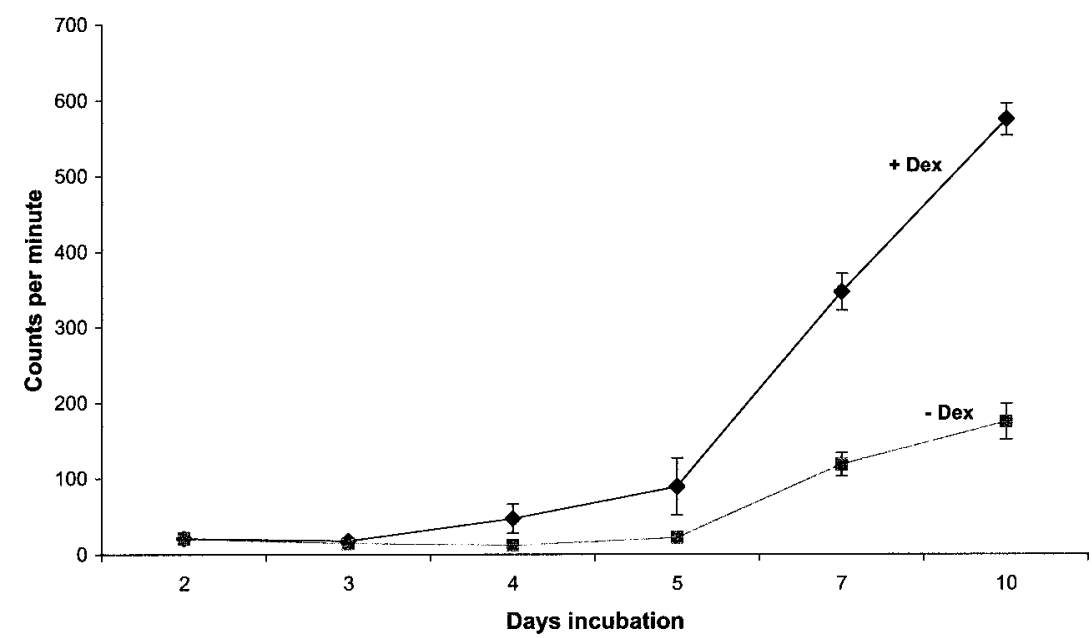

Figure 2 Effect of dexamethasone (Dex) on proliferation of PBMCs as evaluated by $\left[{ }^{3} \mathrm{H}\right]$ thymidine incorporation. Cultures were maintained in the presence $(+)$ of $25 \mathrm{ng} / \mathrm{ml}$ $\mathrm{M}-\mathrm{CSF}, 30 \mathrm{ng} / \mathrm{ml}$ RANKL $\pm 10^{-8} \mathrm{M}$ Dex for various periods of time, after which $\left[{ }^{3} \mathrm{H}\right]$ thymidine incorporation was assessed. Data are expressed as mean \pm S.E.M. of three individual experiments.

\section{Statistical analysis}

Each series of experiments was performed in triplicate, and the data are presented as the mean number of $\mathrm{TRAP}^{+}$ MNCs or mean percentage area lacunar resorption \pm S.E.M. For the purpose of analysis, percentage data were normalised by arcsine transformation. Significant differences were determined using the two-tailed Student's $t$-test; a $P$ value $<0 \cdot 05$ was considered as significant.

\section{Results}

Osteoclast formation and bone resorption in monocyte cultures

Isolated PBMCs cultured on coverslips showed the phenotypic profile of monocytes and did not express osteoclast phenotypic markers. More than $80 \%$ of these cells were CD14 ${ }^{+}$and all were negative for the osteoclast markers TRAP and VNR after $24 \mathrm{~h}$ of incubation. No evidence of lacunar resorption was noted in 24-h cultures of PBMCs on dentine slices. PBMCs cultured with M-CSF and RANKL, in both the presence and the absence of dexamethasone, differentiated into osteoclasts, as demonstrated by the formation of numerous $\mathrm{TRAP}^{+}$ MNCs in 14-day cultures on coverslips and the production of lacunar resorption pits in 21-day cultures on dentine slices (Fig. 1). MNCs formed in PBMC cultures to which dexamethasone was added were generally larger and contained more nuclei than those in the cultures to which no dexamethasone had been added.

\section{Analysis of the various stages of osteoclast formation from circulating precursors}

The proliferative activity of osteoclast precursors in PBMC cultures incubated with RANKL and M-CSF was evaluated by adding hydroxyurea for various intervals of the 21-day culture period and determining the effect on the formation of $\mathrm{TRAP}^{+} \mathrm{MNCs}$ and lacunar resorption (Tanaka et al. 1993). It was found that the addition of hydroxyurea either throughout the 21-day incubation period or during the first 7 days of PBMC culture completely inhibited the formation of $\mathrm{TRAP}^{+} \mathrm{MNCs}$ and the production of lacunar resorption pits (Table 1). Lacunar resorption was also markedly reduced when hydroxyurea was added for the first 11 and 14 days of culture. In contrast, if hydroxyurea was added 17 days after the commencement of cultures, there was no effect on lacunar resorption relative to control cultures to which hydroxyurea was not added (Table 1). This was reflected by an increase in the formation of $\mathrm{TRAP}^{+}$MNCs. $\left[{ }^{3} \mathrm{H}\right]$ Thymidine incorporation also occurred predominantly in the first 7-10 days of PBMC culture, with maximal incorporation seen at 7 days of culture (Fig. 2). These findings indicate that the proliferation phase of human osteoclast formation from circulating precursors occurs predominantly in the first 7-10 days of PBMC culture (Fig. 2).

To analyse further the post-proliferation phase of human osteoclast formation, during which osteoclast precursors undergo further differentiation into cells capable of lacunar bone resorption, we incubated PBMC cultures for 7, 10, 14, 17 and 21 days and then determined when $\mathrm{TRAP}^{+}$MNCs and resorption pit formation first appeared in these cultures. A few $\mathrm{TRAP}^{+} \mathrm{MNCs}$ were noted after 10 days of incubation in some PBMC cultures; their number was considerably more, however, in PBMC cultures incubated for 14 or more days (Fig. 3a). A few lacunar resorption pits were seen in some, but not all, PBMC cultures after 10 days of incubation 


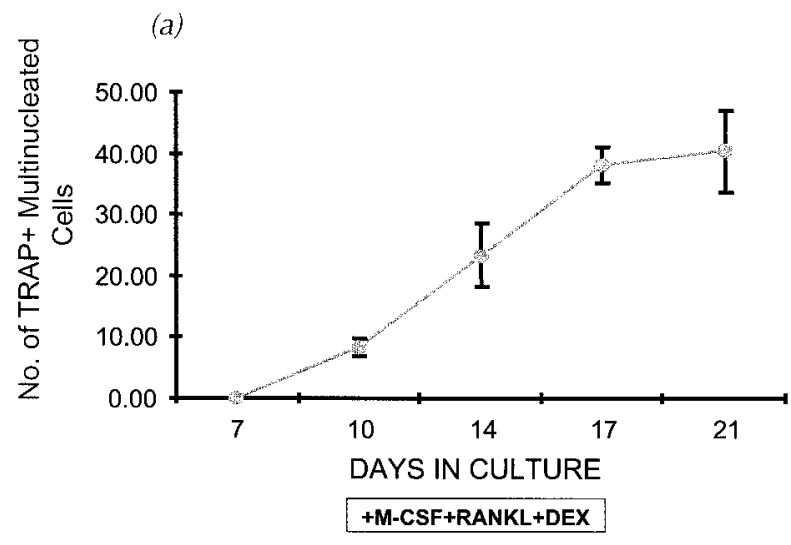

(b)

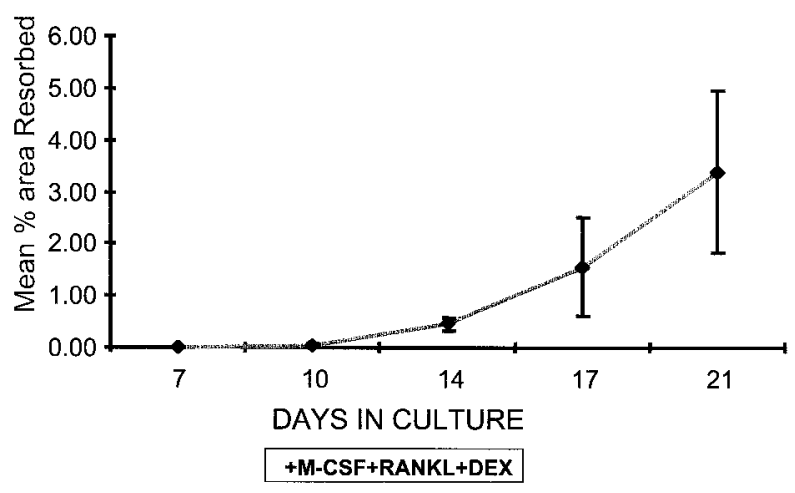

Figure 3 Time-course study of osteoclast formation and lacunar resorption in PBMC cultures incubated up to 21 days in the presence of sRANKL $(30 \mathrm{ng} / \mathrm{ml}), \mathrm{M}-\mathrm{CSF}(25 \mathrm{ng} / \mathrm{ml})$ and Dex $\left(10^{-8} \mathrm{M}\right)$. (a) Mean \pm S.E.M. number of TRAP ${ }^{+}$MNCs formed in cultures. (b) Mean \pm S.E.M. percentage surface area lacunar resorption in cultures.

(mean percentage lacunar resorption: $0.04 \pm 0.02 \%$ ). Considerably more resorption, however, was noted in all PBMC cultures incubated for 14, 17 and 21 days (Fig. 3b). These results indicate that osteoclast activation (i.e. lacunar resorption) occurs mainly after 14 days incubation of circulating osteoclast precursors.

\section{Effect of dexamethasone on different stages of osteoclast} formation from circulating precursors

As assessed by the formation of $\mathrm{TRAP}^{+} \mathrm{MNCs}$ and the extent of lacunar resorption, the addition of dexamethasone to PBMC cultures for either the first 7 or first 14 days of incubation resulted in a significant increase in osteoclast formation relative to that in control cultures to which no dexamethasone had been added (Fig. 4, Table 2). The addition of dexamethasone in the final week of culture alone (i.e. 14-21 days) resulted in a reduction of the extent of lacunar resorption as compared with cultures to which no dexamethasone had been added (69\% compared with
100\%; Fig. 4), this effect was not found to be statistically significantly different. In general, dexamethasone stimulation of osteoclast formation appeared to predominate, as the addition of dexamethasone throughout the 21-day culture period resulted in a significant increase in lacunar resorption compared with that in control cultures to which no dexamethasone had been added (Fig. 4). Increasing the concentration of dexamethasone added to PBMC cultures over this 21-day culture period resulted in a dosedependent increase in the extent of lacunar resorption (Fig. 5).

\section{Bone-resorbing activity of osteoclasts isolated from giant cell tumour of bone}

To ascertain further the effect of dexamethasone on the bone-resorbing activity of mature osteoclasts, these cells were isolated and cultured from two giant cell tumours of bone in the presence and absence of dexamethasone for 24 h. Dexamethasone significantly inhibited the boneresorbing activity of mature osteoclasts. The mean percentage area lacunar resorption in giant cell cultures incubated in the presence of dexamethasone for $24 \mathrm{~h}$ was significantly less $(0.3 \pm 0.01 \%)$ than that in the cultures with no added dexamethasone $(1 \cdot 4 \pm 0 \cdot 08 ; P=0 \cdot 02)$.

\section{Discussion}

Osteoporosis is a common and serious complication of systemic corticosteroid treatment. Corticosteroids are known to influence bone metabolism, but the precise mechanism by which they decrease bone mass is unknown. A role for increased osteoclast formation in the pathogenesis of steroid-associated osteoporosis is suggested by the fact that an increase in osteoclast numbers is commonly seen in bone biopsies of patients who are affected by this condition (Bressot et al. 1979, Dempster 1989). Although secondary hyperparathyroidism (as a result of the effects of steroid on calcium absorption and urinary excretion) may account for some of the increase in osteoclast numbers in bone that occurs after steroid treatment (Lukert \& Adams 1976), the results of cell culture studies indicate that corticosteroids also influence osteoclast formation and activity. Our analysis of the direct effect of dexamethasone on human osteoclast formation and lacunar resorption suggests that corticosteroids stimulate the proliferation and differentiation of circulating osteoclast precursors, but inhibit the bone-resorbing activity of mature osteoclasts.

Glucocorticoid receptors have been identified on both monocytes and bone stromal cells, including osteoblastlike cells (Fieldman et al. 1975, Werb et al. 1978). It has been shown that the addition of dexamethasone to mouse and human marrow cultures, including primary human marrow cultures and cocultures of bone marrow and 


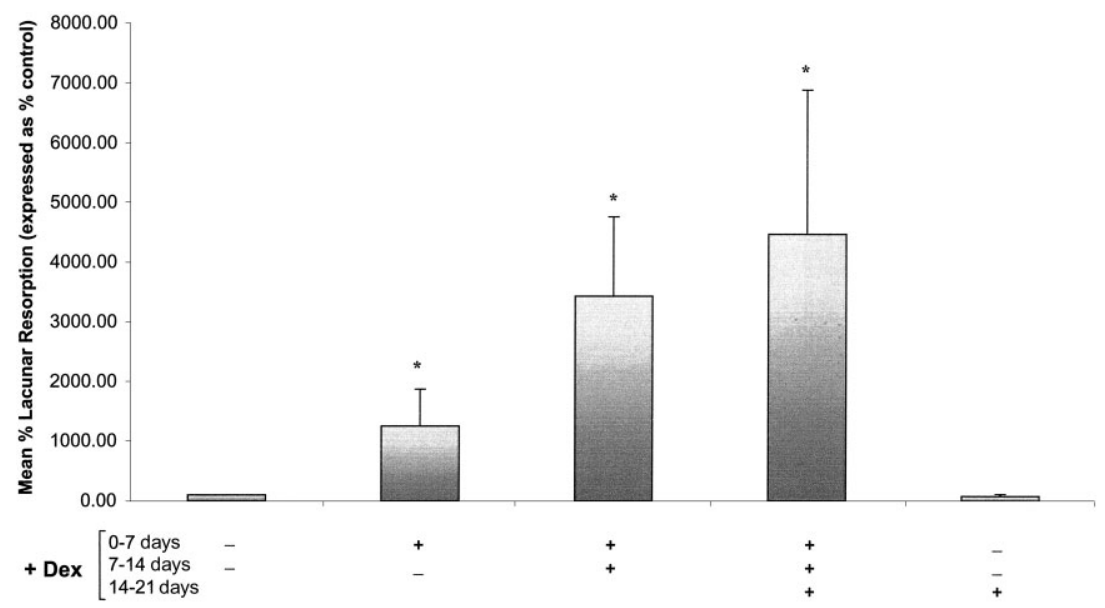

Figure 4 Effect of Dex on RANKL-induced lacunar resorption by osteoclasts formed in PBMC cultures. The addition of Dex $\left(10^{-8} \mathrm{M}\right)$ to the human PBMC cell cultures was omitted at various time points during the 21-day incubation. The 21-day incubation period was divided into 3-week intervals during which dexamethasone was either added to (+) or omitted from $(-)$ the cultures. The mean percentage surface area resorption on three or four dentine slices was calculated and the results were expressed as a percentage of the control cultures to which no Dex had been added ( \pm S.E.M.). All cultures had $30 \mathrm{ng} / \mathrm{ml}$ sRANKL and $25 \mathrm{ng} / \mathrm{ml}$ M-CSF added throughout the 21 days. ${ }^{*} P<0.05$ compared with control cultures.

Table 2 Effect of dexamethasone on PBMC cultures containing sRANKL $(30 \mathrm{ng} / \mathrm{ml})$ and M-CSF $(25 \mathrm{ng} / \mathrm{ml})$

\begin{tabular}{|c|c|c|c|c|}
\hline & \multicolumn{2}{|c|}{ TRAP $^{+}$MNCs (No.) } & \multicolumn{2}{|c|}{ Area resorbed (\%) } \\
\hline & Mean \pm S.E.M. & $\%$ Controlt & Mean \pm S.E.M. & $\%$ Control† \\
\hline \multicolumn{5}{|l|}{ Treatment } \\
\hline No dexamethasone & $24 \cdot 3 \pm 14 \cdot 8$ & 100 & $0 \cdot 263 \pm 0 \cdot 12$ & 100 \\
\hline Dexamethasone & $48 \pm 12 \cdot 1^{*}$ & $169 \pm 34 \cdot 2^{*}$ & $1 \cdot 62 \pm 0 \cdot 36^{*}$ & $4468 \cdot 8 \pm 2410^{*}$ \\
\hline
\end{tabular}

bone-derived stromal cells, results in an increase in osteoclast formation and bone resorption (Van de Wijngaert et al. 1987, Udagawa et al. 1989, Kaji et al. 1997). As various cell types are present in these marrow cultures, it is not certain whether this glucocorticoid enhancement of osteoclast formation is primarily due to an effect on bone stromal cells or one on osteoclast precursors. Dexamethasone is known to promote the expression of osteoclastogenic factors, including interleukin-6 receptor and membrane-bound $\mathrm{M}-\mathrm{CSF}$, in mouse osteoblastlike cells (Udagawa et al. 1995, Rubin et al. 1998). Glucocorticoids also downregulate the expression of osteoprotegerin and stimulate RANKL expression in human osteoblast-like cells (Vidal et al. 1998, Hofbauer et al. 1999).
An advantage of the system of osteoclastogenesis used in this study is that osteoclasts are formed from cells of the monocyte fraction alone (i.e. in the absence of stromal cells); this permits a direct effect of glucocorticoids on osteoclast formation and resorption to be identified. To analyse this effect in more detail, in this study we first established that osteoclast formation from circulating precursors involves an early phase of proliferation of osteoclast progenitors and a later phase in which these cells differentiate into mature bone-resorbing osteoclasts. $\left[{ }^{3} \mathrm{H}\right]$ Thymidine incorporation in PBMC cultures was maximal at day 7 . Addition of hydroxyurea in the first 7 days, but not the final 14 days, of culture resulted in complete inhibition of the formation of $\mathrm{TRAP}^{+} \mathrm{MNCs}$ and lacunar resorption. The addition of hydroxyurea in the 


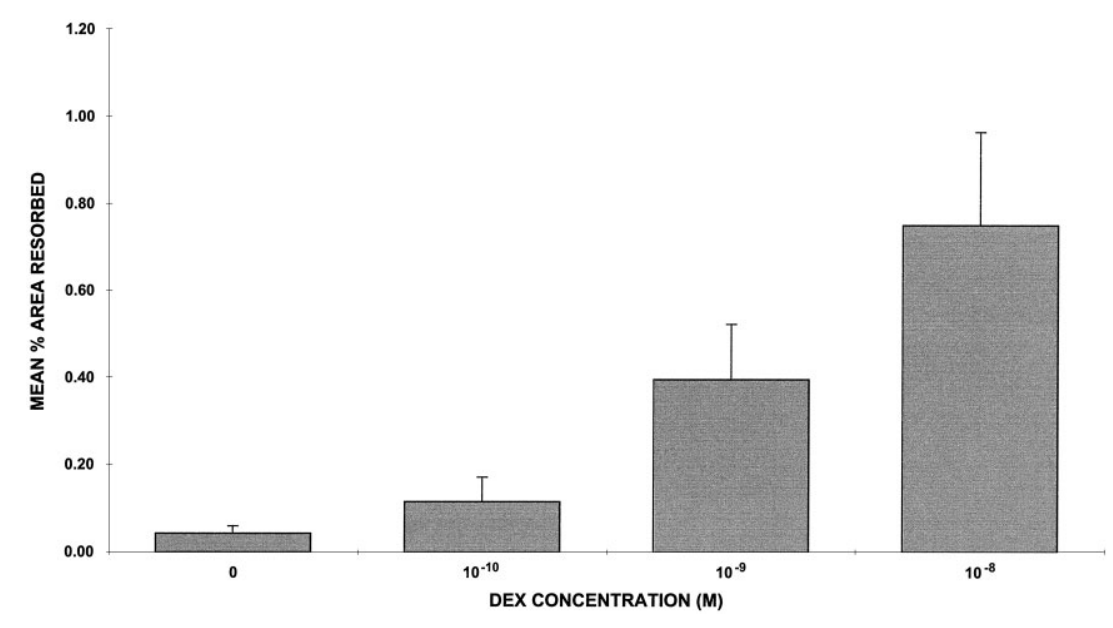

Figure 5 Effect of Dex $\left(0,10^{-10}, 10^{-9}, 10^{-8} \mathrm{M}\right)$ on lacunar resorption in PBMC cultures after 21 days of incubation in the presence of sRANKL $(30 \mathrm{ng} / \mathrm{ml})$ and M-CSF $(25 \mathrm{ng} / \mathrm{ml})$. The percentage data are expressed as the transformed value and are represented as the mean percentage area resorbed \pm S.E.M.

first 10 days of culture resulted in a 30\% decrease in the extent of lacunar resorption. These findings indicate that cell proliferation in PBMC cultures occurs predominantly in the first 7-10 days of PBMC culture. TRAP ${ }^{+}$MNCs and lacunar resorption were first noted after 7 and 10 days of PBMC culture respectively, and the extent of lacunar resorption markedly increased in the final week (i.e. 14-21 days) of culture, indicating that activation (i.e. bone resorption) by osteoclasts formed in these cultures occurs predominantly at this time. Although it is clear that these two phases overlap, these findings permit the effects of dexamethasone on human osteoclast precursor proliferation and osteoclast activation to be assessed separately.

The addition of dexamethasone to PBMC cultures for either the first 7 or the first 14 days of incubation resulted in a significant increase in the formation of $\mathrm{TRAP}^{+}$ MNCs capable of extensive lacunar resorption, indicating that dexamethasone promotes the proliferation and differentiation of osteoclast precursors. The addition of dexamethasone in the final week of culture alone (i.e. the period during which most lacunar resorption occurs) resulted in an insignificant decrease in the extent of lacunar resorption. Although the manner in which glucocorticoids promote the proliferation and differentiation of human osteoclast precursors is uncertain (Itonaga et al. 1999), these compounds in general act by enhancing the effect of other humoral factors. It has previously been shown that, in the presence of dexamethasone and $1,25(\mathrm{OH})_{2} \mathrm{D}_{3}$, osteoclast progenitor cells show enhanced expression of RANK (Nagai \& Sato 1999). It is thus possible that, in our monocyte cultures, dexamethasone is acting directly to increase the expression of RANK on circulating osteoclast precursors; alternatively, it could promote monocyte production of one or more humoral factors that increase RANK expression. As glucocorticoids are also known to modulate the activation of NF- $\kappa B$, it is possible that, either directly or indirectly (e.g. via cytokines), glucocorticoid-induced changes in NF- $\mathrm{KB}$ activition promote RANKL/M-CSF-induced osteoclast formation (Auphan et al. 1995).

Previous studies of the effect of glucocorticoids on osteoclast activity and bone resorption have provided contradictory results. Glucocorticoids have been reported to inhibit bone resorption in fetal rat long bone cultures, but to increase resorption in mouse calvarial cultures (Teitelbaum et al. 1981, Reid et al. 1986). Glucocorticoids have also been found to exert dose-dependent inhibition of lacunar bone resorption by rat osteoclasts (Tobias \& Chambers 1989). Our results would also indicate that corticosteroids inhibit the bone-resorbing activity of mature osteoclasts. We found that the addition of dexamethasone during the third week of PBMC culture resulted in an insignificant reduction in lacunar resorption by the osteoclasts that formed in these cultures. To confirm these findings, we further noted that dexamethasone inhibited lacunar resorption by osteoclasts isolated from two giant cell tumours of bone. Although the mechanism whereby dexamethasone inhibits the boneresorbing activity of mature osteoclasts is unknown, it is possible, as previous studies have indicated, that corticosteroids inhibit lacunar resorption by increasing osteoclast apoptosis (Tobias \& Chambers 1989, Dempster et al. 1997).

Our results indicate that the effect of glucocorticoids on osteoclasts is biphasic. There is stimulation of osteoclast formation through promotion of proliferation and differentiation of osteoclast precursors to $\mathrm{TRAP}^{+} \mathrm{MNCs,}$ 
but inhibition of the bone-resorbing activity of mature osteoclasts. These findings suggest that the increase in osteoclast numbers associated with the osteopenia of longterm corticosteroid use is primarily the result of an increase in the proliferation and differentiation of osteoclast precursors. It would appear that this effect predominates over that of inhibition of the bone-resorbing activity of mature osteoclasts. If this is the case, then the use of therapeutic agents that inhibit osteoclast formation (e.g. osteoprotegerin) may be effective in the treatment of corticosteroid-induced osteoporosis.

\section{Acknowledgements}

This work was carried out with the funding support of The Wellcome Trust, the Nuffield Foundation and Action Research. The authors wish to thank Amgen Inc. (USA) for supplying soluble RANKL.

\section{References}

Adachi JD 1997 Corticosteroid-induced osteoporosis. American Journal of Medical Science 313 41-49.

Athanasou NA \& Quinn JM 1990 Immunophenotypic differences between osteoclasts and macrophage polykaryons: immunohistological distinction and implications for osteoclast ontogeny and function. Journal of Clinical Pathology 43 997-1004.

Auphan N, DiDonato JA, Rosette C, Helmberg A \& Karin M 1995 Immunosuppression by glucocorticoids: inhibition of NF- $\mathrm{KB}$ activity through induction of IאB synthesis. Science 270 286-290.

Boyde A, Ali N \& Jones SJ 1984 Resorption of dentine by isolated osteoclasts in vitro. British Dental Journal 156 216-220.

Bressot CP, Meunier J, Lejeune E, Edouard C \& Darby AJ 1979 Histomorphometric profile, pathophysiology and reversibility of glucocorticoid-induced osteoporosis. Metabolic Bone Disease and Related Research 1 303-311.

Chambers TJ, Fuller K, McSheehy PM \& Pringle JA 1985 The effects of calcium regulating hormones on resorption by isolated human osteoclastoma cells. Journal of Pathology 145 297-305.

Dempster DW 1989 A perspective: bone histomorphometry in glucocorticoid-induced osteoporosis. Journal of Bone and Mineral Research 4 137-141.

Dempster DW, Moonga BS, Stein LS, Horbert WR \& Antakly T 1997 Glucocorticoids inhibit bone resorption by isolated rat osteoclasts by enhancing apoptosis. Journal of Endocrinology 154 397-406.

Eastell R, Reid DM, Compston J, Cooper C, Fogelman I, Francis RM, Hosking DJ, Purdie DW, Ralston SH, Reeve J, Russell RG, Stevenson JC \& Torgerson DJ 1998 A UK consensus group on management of glucocorticoid-induced osteoporosis: an update. Journal of Internal Medicine 244 271-292.

Fieldman D, Dziak R, Koehler R \& Stern P 1975 Cytoplasmic glucocorticoid binding in bone cells. Endocrinology 96 29-36.

Fujikawa Y, Quinn JMW, Sabokbar A, McGee JOD \& Athanasou NA 1996 The human osteoclast precursor circulates in the monocyte fraction. Endocrinology 137 4058-4060.

Hofbauer LC, Gori F, Riggs BL, Lacey DL, Dunstan CR, Spelsberg TC \& Khosla S 1999 Stimulation of osteoprotegerin ligand and inhibition of osteoprotegerin production by glucocorticoids in human osteoblastic lineage cells: potential paracrine mechanisms of glucocorticoid-induced osteoporosis. Endocrinology 140 4382-4392.
Horton MA, Lewis D, McNulty K, Pringle JAS \& Chambers TJ 1985 Monoclonal antibodies for osteoclastomas (giant cell bone tumors): identification of osteoclast-specific antigens. Cancer Research $\mathbf{4 5}$ $5663-5669$

Itonaga I, Sabokbar A, Neale SD \& Athanasou NA 1999 1,25Dihydroxyvitamin $\mathrm{D}_{3}$ and prostaglandin $\mathrm{E}_{2}$ act directly on circulating human osteoclast precursors. Biochemical and Biophysical Research Communications 264 590-595.

Kaji H, Sugimoto T, Kanatani M, Nishiyama K \& Chihara K 1997 Dexamethasone stimulates osteoclast-like cell formation by directly acting on hemopoietic blast cells and enhances osteoclast-like formation stimulated by parathyroid hormone and prostaglandin E2. Journal of Bone and Mineral Research 12 734-741.

Lukert BP \& Adams JS 1976 Calcium and phosphorus homeostasis in man: effect of corticosteroids. Archives of Internal Medicine $\mathbf{1 3 6}$ $1249-1253$

Manolagas SC 2000 Corticosteroids and fractures: a close encounter of the third cell kind. Journal of Bone Mineral Research 15 1001-1005.

Matsuzaki K, Udagawa N, Takahashi N, Yamaguchi K, Yasuda H, Shima N, Morinaga T, Toyama Y, Yabe Y, Higashio K \& Suda T 1998 Osteoclast differentiation factor (ODF) induces osteoclast-like cell formation in human peripheral blood mononuclear cell cultures. Biochemical and Biophysical Research Communications 246 199-204.

Minkin C 1982 Bone acid phosphatase: tartrate-resistant acid phosphatase as a marker of osteoclast function. Calcified Tissue International 34 285-290.

Nagai M \& Sato N 1999 Reciprocal gene expression of osteoclastogenesis inhibitory factor and osteoclast differentiation factor regulates osteoclast formation. Biochemical and Biophysical Research Communications 257 719-723.

Nishimura J \& Ikuyama S 2000 Glucocorticoid-induced osteoporosis: pathogenesis and management. Journal of Bone and Mineral Metabolism 18 350-352.

Quinn JMW, Elliott J, Gillespie MT \& Martin TJ 1998 A combination of osteoclast differentiation factor and macrophagecolony stimulating factor is sufficient for both human and mouse osteoclast formation. Endocrinology 139 4424-4427.

Raisz LG, Trummel CL, Wener JA \& Simmons H 1972. Effect of glucocorticoids on bone resorption in tissue culture. Endocrinology $\mathbf{9 0}$ 961-967.

Reid IR, Katz JM, Ibbertson HK \& Gray DH 1986 The effects of hydrocortisone, parathyroid hormone and the bisphosphonate APD on bone resorption in neonatal mouse calvaria. Calcified Tissue International 38 38-43.

Rubin J, Biskobing DM, Jadhav L, Fan MS, Nanes MS, Perkins S \& Fan X 1998 Dexamethasone promotes expression of membranebound macrophage colony-stimulating factor in murine osteoblastlike cells. Endocrinology 139 106-112.

Tanaka S, Takahashi N, Udagawa N, Tamura T, Akatsu T, Stanley ER, Kurokawa T \& Suda T 1993 Macrophage colony-stimulating factor is indispensable for both proliferation and differentiation of osteoclast progenitors Journal of Clinical Investigation 91 257-263.

Teitelbaum S, Malone JD \& Kahn AJ 1981. Glucocorticoid enhancement of bone resorption by rat peritoneal macrophages in vitro. Endocrinology 108 795-799.

Tobias J \& Chambers TJ 1989 Glucocorticoids impair bone resorptive activity and viability of osteoclasts disaggregated from neonatal rat long bones. Endocrinology 125 1290-1296.

Udagawa N, Takahashi N, Akatsu T, Sasaki T, Yamaguchi A, Kodama H, Martin TJ \& Suda T 1989 The bone marrow-derived stromal cells lines MC3T3-G2/PA6 and ST2 support osteoclast-like cell differentiation in cocultures with mouse spleen cells. Endocrinology 125 1805-1813.

Udagawa N, Takahashi N, Katagiri T, Tamura T, Wada S, Findlay DM, Martin TJ, Hirota H, Taga T, Kishimoto T \& Suda T 1995 IL-6 induction of osteoclast differentiation depends on IL-6 receptors expressed in osteoblastic cells but not on osteoclast progenitors. Journal of Experimental Medicine 182 1461-1468. 
Van de Wijngaert P, Taws M, Vandermeer J \& Burger E 1987 Growth of osteoclast precursor-like cells from whole bone marrow. Bone and Mineral 3 97-110.

Van Staa T, Leufkens H, Abenhaim L, Zhang B \& Cooper C 2000 Use of oral corticosteroids and risk of fractures. Journal of Bone Mineral Research 15 993-1000.

Vidal N, Brändström H, Jonsson KB \& Ohlsson C 1998 Osteoprotegerin mRNA is expressed in primary human osteoblastlike cells: down-regulation by glucocorticoids. Journal of Clinical Investigation 159 191-195.

Werb Z, Foley R \& Munck A 1978 Interaction of glucocorticoids with macrophages. Identification of glucocorticoid receptor in monocytes and macrophages. Journal of Experimental Medicine 147 1684-1694.

Yasuda H, Shima N, Nakagawa N, Yamaguchi K, Kinosaki M, Mochizuki S-I, Tomoyasu A, Yano K, Goto M, Murakami A, Tsuda E, Morinaga T, Higashio K, Udagawa N, Takahashi N \& Suda T 1998 Osteoclast differentiation factor is a ligand for osteoprotegerin/osteoclastogenesis-inhibitory factor and is identical to TRANCE/RANKL. PNAS 95 3597-3602.

Received 6 June 2002

Accepted 12 June 2002 